\title{
A Cross-sectional Survey of Chronic Kidney Disease and Diabetic Kidney Disease in Japanese Type 2 Diabetic Patients at Four Urban Diabetes Clinics
}

\author{
Yuko Watanabe ${ }^{1,2}$, Hitomi Fujii ${ }^{1,3}$, Kanemi Aoki ${ }^{4}$, Yasuhiko Kanazawa ${ }^{4}$ \\ and Takaichi Miyakawa ${ }^{1}$
}

\begin{abstract}
Objective Management of chronic kidney disease (CKD) is a critical issue in public health in attempt to prevent kidney failure and dialysis dependency. Since 1998, diabetes mellitus has been the leading cause of dialysis dependency in Japan. Previous reports demonstrated that the prevalence of CKD in diabetic patients was high; however albuminuria was not always present. This cross-sectional survey was performed 1) to indicate the prevalence of CKD and co-morbid illness in diabetic patients seen at diabetic clinics, and 2) to demonstrate the relationship between estimated glomerular filtration rate (eGFR) and urine albumin to creatinine ratio (ACR).

Patients and Methods A total of 288 consecutive adult type 2 diabetic patients seen at four diabetic clinics in the Tokyo Metropolitan Area were enrolled in November 2007. We excluded patients with kidney failure. Estimated GFR was calculated by the MDRD Study equation with the Japanese coefficient.

Results Patients had a mean age (+/- SD), $61(+/-12)$; male, 58\%; mean BMI, $25.2 \mathrm{~kg} / \mathrm{m}^{2}(+/-5.2)$; and mean $\mathrm{HbA}_{\mathrm{lc}}, 7.1 \%$ (+/- 1.3). The prevalence of CKD stage 3 was 38\% (109/288) with 64\% (70/109) of them being normoalbuminuric. Co-morbid illnesses, including hypertension $(\mathrm{p}<0.001)$ and old stroke $(\mathrm{p}=0.02)$, were significantly higher in CKD stage 3 patients.

Conclusion Our patients were relatively young and obese, reflecting urban clinical settings. The prevalence of CKD stage 3 patients was high. Clinicians need to check both eGFR and ACR to avoid underdiagnosis of CKD and diabetic kidney disease.
\end{abstract}

Key words: albumin to creatinine ratio (ACR), chronic kidney disease (CKD), diabetic kidney disease (DKD), type 2 diabetes mellitus, modification of diet in renal disease (MDRD) study equation

(Inter Med 48: 411-414, 2009)

(DOI: 10.2169/internalmedicine.48.1691)

\section{Introduction}

Management of chronic kidney disease (CKD) is a critical issue in public health for preventing kidney failure and dialysis dependency. In Japan, the prevalence of CKD stage 3, defined as an estimated glomerular filtration rate (eGFR) of between 30 and $59 \mathrm{~mL} / \mathrm{min} / 1.73 \mathrm{~m}^{2}$, was $18.5 \%$ in the general adult population in 2007 , which is higher than western countries such as the USA and the UK $(1,2)$. The main rea- sons were the high prevalence of kidney disease, including chronic glomerulonephritis, hypertensive nephrosclerosis, and polycystic kidney disease; and the growing elderly population in Japan. Furthermore, in Japan, diabetes mellitus has become the leading cause of dialysis dependence since 1998. In 2006, 15,000 diabetic patients became dialysis dependent, which accounted for $40 \%$ of new dialysis patients (3).

Diabetic kidney disease (DKD) is a presumptive diagnosis of kidney disease caused by diabetes, and in clinical set-

${ }^{1}$ Department of Internal Medicine, Tama-center Mirai Clinic, Tama, ${ }^{2}$ Department of Internal Medicine, Ofuna Chuo Hospital, Kamakura, ${ }^{3}$ Department of Internal Medicine, Nakano Kyoritsu Clinic, Tokyo and ${ }^{4}$ Department of Internal Medicine, JA Isehara Kyodo Hospital, Isehara Received for publication September 16, 2008; Accepted for publication November 27, 2008 Correspondence to Dr. Yuko Watanabe, yukow@shonan.ne.jp 
tings, the presence of albuminuria is essential for diagnosis, although the gold standard is pathological confirmation by kidney biopsy (4). The extent of albuminuria is divided into two groups: microalbuminuria [albumin to creatinine ratio (ACR) 30 299 mg/g creatinine] and macroalbuminuria (ACR $>300 \mathrm{mg} / \mathrm{g}$ creatinine). In the typical clinical course of DKD, eGFR remains normal or elevated in the microalbuminuric state, and then declines progressively, especially in the late macroalbuminuric state. DKD staging by the Japan Diabetes Society requires both GFR and ACR (5); however, an increase in ACR does not always accompany with a decrease in GFR in type 2 diabetic patients with DKD. For example, the Third National Health and Nutrition Examination Study showed that in type 2 diabetic patients with GFR $<60 \mathrm{~mL} / \mathrm{min} / 1.73 \mathrm{~m}^{2}$, retinopathy and albuminuria were both absent in $30 \%$ of the study population (6). This study indicated that a substantial number of type 2 diabetic patients had decreased GFR, secondary to other glomerular, or interstitial lesions, such as ischemic nephropathy, rather than diabetic nephropathy (7).

To our knowledge, few studies have reported the relationship between CKD and albuminuria in Japanese type 2 diabetic patients $(8,9)$. Also, clinical information for normoalbuminuric diabetic patients with CKD is lacking. This crosssectional survey was performed 1) to ascertain the prevalence of CKD and co-morbid illness in our diabetic clinic patients, and 2) to demonstrate the relationship between eGFR and ACR.

\section{Patients and Methods}

A total of 288 consecutive adult type 2 diabetic patients seen at four diabetic clinics in the Tokyo Metropolitan Area in November 2007 were enrolled. Eligible patients had serum creatinine and spot urine ACR measured within six months. We excluded patients with kidney failure whose GFR was less than $15 \mathrm{~mL} / \mathrm{min} / 1.73 \mathrm{~m}^{2}$. There was no dialysis patient.

The definition of CKD by the Japanese Society of Nephrology is an eGFR of less than $60 \mathrm{~mL} / \mathrm{min} / 1.73 \mathrm{~m}^{2}$ and/or an evidence of kidney damage such as proteinuria or hematuria lasting for at least 3 months. Estimated GFR was calculated by the Modification of Diet in Renal Disease (MDRD) study equation using a coefficient for Japanese. The equation has been revised in May 2008 as follows: eGFR $\left(\mathrm{mL} / \mathrm{min} / 1.73 \mathrm{~m}^{2}\right)=0.808 \times 175 \times \mathrm{Cr}^{-1.154} \times \mathrm{Age}^{-0.203} \times 0.742$ (if female) (10).

DKD was defined by increased urine albumin secretion based on an ACR, which is divided into microalbuminuria and macroalbuminuria. In this survey, ACR was measured at least once by immunoturbidimetric assay; and serum creatinine, at least twice by the enzyme method within the previous six months. The Japan Diabetes Society defined DKD staging I through $\mathrm{V}$ according to ACR and eGFR as follows. Stage I: ACR $<30 \mathrm{mg} / \mathrm{g}$ creatinine and normal or high in eGFR; stage II: ACR 30 299 mg/g creatinine and normal or high in eGFR; stage III: ACR $>300 \mathrm{mg} / \mathrm{g}$ creatinine or persistent proteinuria with decreased eGFR; stage IV: kidney failure; and stage V: dialysis dependency (5).

Co-morbid illness was defined as positive if complying with each disease criteria as follows or having been on treatment: dyslipidemia, fasting low density lipoprotein greater than $140 \mathrm{mg} / \mathrm{dL}$ and/or fasting triglyceride greater than 150 $\mathrm{mg} / \mathrm{dL}$; hypertension, systolic blood pressure greater than $140 \mathrm{~mm} \mathrm{Hg}$ and/or diastolic blood pressure greater than 90; and hyperuricemia, serum uric acid greater than $7 \mathrm{mg} / \mathrm{dL}$ or having a history of gout. Positive history of old myocardial infarction was confirmed either by pathological Q waves on electrocardiogram or by history of coronary intervention and/or bypass surgery. Old stroke was defined as having history of any cerebral or cellebelar infarction or hemorrhage.

Statistical analyses were performed by the $\mathrm{JMP}^{\circledR} 6.0$ using Statistical Analysis System. We performed group comparisons using chi-square test for categorical data and one way ANOVA for continuous data.

\section{Results}

In this survey, 288 type 2 diabetic patients had a mean age (+/- SD), 61 (+/- 12); male, 58\%; mean BMI, 25.2 $\mathrm{kg} / \mathrm{m}^{2}$ (+/- 5.2); mean $\mathrm{HbA}_{\mathrm{cc}}, 7.1 \%$ (+/- 1.3); and more than 10 years of diabetes duration, $48 \%$. Patients who had a previous history of myocardial infarction or stroke were $4 \%$ and $8 \%$, respectively.

Patient characteristics were compared according to eGFR strata $\left(30 \sim 59 \mathrm{~mL} / \mathrm{min} / 1.73 \mathrm{~m}^{2}, 60 \sim 89 \mathrm{~mL} / \mathrm{min} / 1.73 \mathrm{~m}^{2}\right.$, greater than $90 \mathrm{~mL} / \mathrm{min} / 1.73 \mathrm{~m}^{2}$ ) and the level of albuminuria [Table 1]. There was no patient with eGFR $<30 \mathrm{~mL} /$ $\min / 1.73 \mathrm{~m}^{2}$. Patients with CKD stage 3 were $37.8 \%$ (109/ $288)$ and they demonstrated significantly lower $\mathrm{HbA}_{\mathrm{lc}}(\mathrm{p}=$ $0.008)$, longer duration of diabetes $(\mathrm{p}=0.001)$, high prevalence of proliferative retinopathy $(\mathrm{p}=0.003)$, hypertension $(\mathrm{p}=0.001)$, and old stroke $(\mathrm{p}=0.02)$, using more ACEIs/ ARBs $(\mathrm{p}=0.001)$ than patients with eGFR $>60 \mathrm{~mL} / \mathrm{min} / 1.73$ $\mathrm{m}^{2}$. As for diabetic treatment, $40 \%$ of patients with eGFR > $90 \mathrm{~mL} / \mathrm{min} / 1.73 \mathrm{~m}^{2}$ were on insulin alone, whereas patients with eGFR $<60 \mathrm{~mL} / \mathrm{min} / 1.73 \mathrm{~m}^{2}$ were more on combination therapy with insulin and oral antidiabetic agents (OADs) $(\mathrm{p}=$ 0.02).

Patient characteristics between normoalbuminuric and albuminuric patients were also compared [Table 1]. Serum creatinine and eGFR did not show a significant difference between the groups. Albuminuric patients demonstrated higher BMI $(\mathrm{p}=0.04)$, higher $\mathrm{HbA}_{\mathrm{lc}}(\mathrm{p}=0.03)$, higher prevalence of dyslipidemia $(\mathrm{p}=0.005)$, hypertension $(\mathrm{p}=0.008)$, and hyperuricemia $(\mathrm{p}<0.001)$; and they were prescribed more ACEIs or ARBs $(p=0.003)$ than normoalbuminuric patients.

Table 2 demonstrates the relationship between eGFR and ACR. In eGFR $>90 \mathrm{~mL} / \mathrm{min} / 1.73 \mathrm{~m}^{2}$ strata, patients with normoalbuminuria were $48 \%(12 / 25)$; patients with microalbuminuria, $48 \%(12 / 25)$; and patients with macroalbumin- 
Table 1. Patients Characteristics according to eGFR Strata and Level of Albuminuria

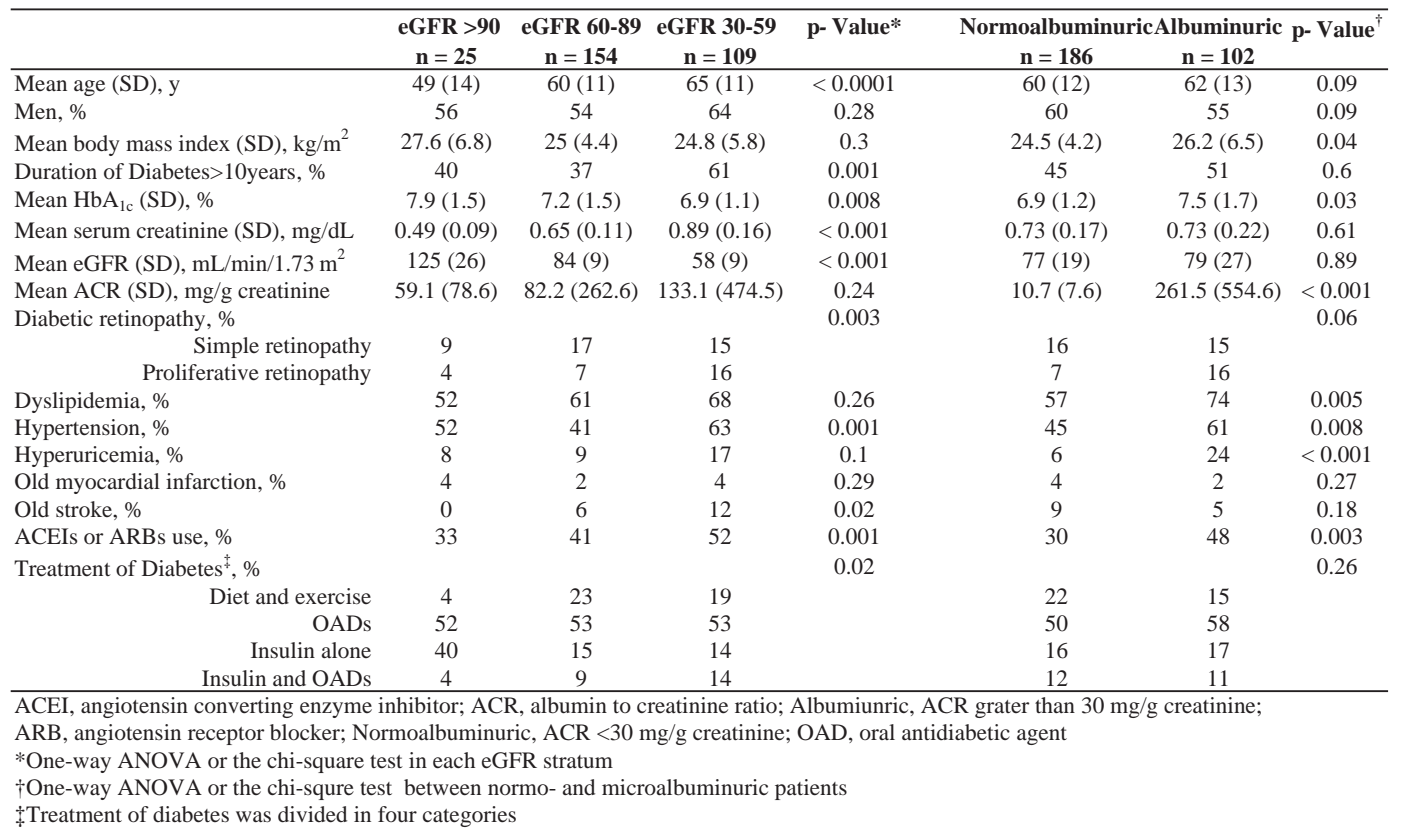

Table 2. Relationship between eGFR and ACR Strata

\begin{tabular}{lcccc}
\hline & eGFR >90 & eGFR 60 89 & eGFR 30 59 & p- Value* \\
& $\mathbf{n}=\mathbf{2 5}$ & $\mathbf{n}=\mathbf{1 5 4}$ & $\mathbf{n = 1 0 9}$ & \\
\hline Normoalbuminuric, $\mathrm{n}(\%)$ & $12(48)$ & $104(68)$ & $70(64)$ & 0.35 \\
Microalbuminuric, $\mathrm{n}(\%)$ & $12(48)$ & $42(27)$ & $32(30)$ & \\
Macroalbuminuric, n $(\%)$ & $1(4)$ & $8(5)$ & $7(6)$ & \\
\hline * The chi-square test between each eGFR and ACR stratum & \\
Normoalbuminuric, ACR <30 mg/g creatinine; Microalbuminuric, ACR 30 299 mg/g creatinine; Macroalbuminuric, ACR $>300 \mathrm{mg} / \mathrm{g}$ creatinin
\end{tabular}

uria, $4 \%$ (1/25); respectively. In eGFR 60 89 $\mathrm{mL} / \mathrm{min} / 1.73$ $\mathrm{m}^{2}$ strata, patients with normoalbuminuria were $68 \%(104 /$ 154); patients with microalbuminuria, 27\% (42/154); and patients with macroalbuminuria, 5\% (8/154); respectively. In eGFR 30 59 mL/min/1.73 $\mathrm{m}^{2}$ strata, patients with normoalbuminuira were $64 \%$ (70/109), patients with microalbuminuria, 30\% (32/109); and patients with macroalbumiuria, $6 \%$ (7/109). There was no significant difference between each eGFR stratum regarding albumin secretion $(\mathrm{p}=0.35)$.

\section{Discussion}

In this survey, patients were relatively young but the prevalence of CKD stage 3 was high. A previous report from the UK (2) revealed that $30 \%$ of diabetic patients had CKD stage 3, which was similar to our result indicating a higher prevalence of CKD in diabetic patients than the general population. Our survey also demonstrated that $64 \%$ of diabetic patients with CKD stage 3 were normoalbuminuric, in contrast to the results which showed that normoalbuminuric patients were about 20 to $30 \%$ in CKD stage 3 patients $(11,12)$. ACEIs and ARBs might have modified both ACR and eGFR, although the absolute number of patients on these drugs in our study were fewer than in the previous studies $(11,12)$. The Japanese DKD staging originally did not take into consideration the discrepancy between changes in eGFR and ACR, especially for normoalbuminuria in CKD stage 3 patients, thus it needs reconsideration.

CKD stage 3 patients in this survey were shown to have more frequent co-morbid illnesses than patients with eGFR greater than $60 \mathrm{~mL} / \mathrm{min} / 1.73 \mathrm{~m}^{2}$ : proliferative retinopathy, hypertension, and old stroke. Compared to the national survey in 2003 regarding diabetic macrovascular complications, patients who had a previous history of myocardial infarction in this survey were less (12\% vs. $4 \%$ ), and patients with stroke were more (4\% vs. $8 \%$ ) (13), which was a prominent character of our study population.

Previous reports indicated that eGFR as determined by the MDRD Study equation might have underestimated elevated or high-normal GFRs in type 2 diabetic patients, whereas detecting GFR less than $60 \mathrm{~mL} / \mathrm{min} / 1.73 \mathrm{~m}^{2}$ was accurate $(1,4,14)$. These results demonstrated that eGFR alone as a screening test for CKD in diabetic patients is insufficient especially in the early years after diagnosis, although elevated eGFR was infrequent in this survey. Furthermore, ACEIs and ARBs treatment may have modified ACR levels, although the mean ACR in each eGFR strata was lower compared to the previous reports $(11,12)$.

Half of the patients in each eGFR stratum were on OADs. 24 to $44 \%$ of each eGFR strata were being treated with either insulin alone or a combination therapy, which was reasonably high since patients were seen at diabetes clinics 
run by diabetologists. Treatment of diabetes showed significant difference between eGFR strata, mainly because $40 \%$ of patients with eGFR $>90 \mathrm{~mL} / \mathrm{min} / 1.73 \mathrm{~m}^{2}$ were on insulin alone, and patients with eGFR $<60 \mathrm{~mL} / \mathrm{min} / 1.73 \mathrm{~m}^{2}$ were treated using a combination of insulin and OADs. Relatively high patient's age and longer duration of diabetes may have altered the physician's decision for diabetic treatment in CKD stage 3 diabetic patients.

There are several limitations in this study. First, this study was a cross-sectional survey at specialist clinics, and the results might not be applicable to clinics of general practioners, thus generalizability was limited to patients with a similar clinical background. Second, this study lacked measurements of GFR by the gold standard such as inulin clearance, although inulin is not useful for routine assessment in clinical settings where inulin clearance is time-consuming and expensive. Finally, $70 \%$ of our patients had urine albumin measurement only once, although urine dip stick test was negative for proteinuria at each visit in normoalbuminuric patients. At least two measurements were required in order to obtain accurate ACR, given that the coefficient of vari- ation of albumin measurements reached $40 \%$ (4).

In conclusion, our patients were relatively young and obese reflecting urban clinical settings; however, the prevalence of CKD stage 3 patients was high and more than $60 \%$ of them showed normoalbuminuria. Clinicians need to check both eGFR and ACR to avoid underdiagnosis of CKD and DKD in type 2 diabetic patients. Further studies are needed to reveal the natural course of normoalbuminuric CKD stage 3 diabetic patients and to determine the causes of CKD in those patients, in order to obtain a complete clinical image of DKD in type 2 diabetic patients.

\section{Acknowledgement}

We presented part of this paper at the 5th World Congress in Prevention of Diabetes and it's complications (WCPD) held in Helsinki, Finland in June 2008.

The authors are indebted to Drs. Germaine Chan and Robert Gray for their editorial work and to Mss. Yoko Ishii, Kasumi Kinebuchi, Ritsuko Kurabayashi, and Sakiko Sato for their work.

\section{References}

1. Imai E, Horio M, Iseki K, et al. Prevalence of chronic kidney disease $(\mathrm{CKD})$ in the Japanese general population predicted by the MDRD equation modified by a Japanese coefficient. Clin Exp Nephrol 11: 156-163, 2007.

2. New JP, Middleton RJ, Klebe B, et al. Assessing the prevalence, monitoring and management of chronic kidney disease in patients with diabetes compared with those without diabetes in general practice. Diabet Med 24: 364-369, 2007.

3. Iseki K. Chronic kidney disease in Japan. Intern Med 47: 681689, 2008.

4. KDOQI. Clinical practice guidelines and clinical practice recommendations for diabetes and chronic kidney disease. Am J Kidney Dis 49: S12-S154, 2007.

5. Haneda M. Diabetic nephropathy: Diagnostic criteria and staging. Nippon Rinsho 63: S320-323, 2005 (in Japanese).

6. Kramer HJ, Nguyen QD, Curhan G, et al. Renal insufficiency in the absence of albuminuria and retinopathy among adults with type 2 diabetes mellitus. JAMA 289: 3273-3277, 2003.

7. Hamano K, Nitta A, Ohtake T, et al. Associations of renal vascular resistance with albuminuria and other macroangiopathy in type 2 diabetic patients. Diabetes Care 31: 1853-1857, 2008.

8. Yokoyama H, Kawai K, Kobayashi M, et al. Microalbuminuria is common in Japanese type 2 diabetic patients. A nationwide survey from Japan Diabetes Clinical Data Management Study Group (JDDM10). Diabetes Care 30: 989-992, 2007.

9. Taniwaki H, Ishimura E, Emoto $M$, et al. Relationship between urinary albumin excretion and glomerular filtration rate in normotensive, nonproteinuric patients with type 2 diabetes mellitus. Nephron 86: 36-43, 2000.

10. Imai E, Horibe M. Evaluation of kidney function. Nippon Naika Gakkai Zasshi 97: 921-928, 2008 (in Japanese).

11. MacIsaac RJ, Smith TJ, Tsalamandris C, et al. Nonalbuminuric renal insufficiency in type 2 diabetes. Diabetes Care 27: 195-200, 2004.

12. Rigalleau V, Barthe N, Lasseur C, et al. Normoalbuminuric renalinsufficiency diabetic patients: A lower-risk group. Diabetes Care 30: 2034-2039, 2007.

13. Diabetes mellitus patients' report 2003 (in Japanese), http:// wwwdbtk.mhlw.go.jp/toukei/kouhyo/indexkk_4_2.html, retrieved August 23, 2008.

14. Levey AS, Coresh J, Greene $\mathrm{T}$, et al. Using standardized serum creatinine values in the modification of diet in renal disease study equation for estimating glomerular filtration rate. Ann Intern Med 145: 247-254, 2006.

(C) 2009 The Japanese Society of Internal Medicine http://www.naika.or.jp/imindex.html 